2 Peng $P W$. Intracranial hypotension with severe neurological symptoms resolved by epidural blood patch. Can J Neurol Sci 2004; 31: 569-71.

3 Arai M, Matsushima S, Terada $H$. Divergence paresis without positional headache: an unusual presentation of cerebrospinal fluid hypovolemia after spinal anesthesia. Anesth Analg 2006; 102: 1865-6.

4 Kilickan L, Gurkan $\Upsilon$, Ozkarakas H. Permanent sensorineural hearing loss following spinal anesthesia. Acta Anaesthesiol Scand 2002; 46: 1155-7.

5 Baloh RW. Vertigo. Lancet 1998; 352: 1841-6.

\section{Neck auscultation: a simple new method for confirming tracheal intubation}

\section{To the Editor:}

I read Dr. Christodolou's recent letter ${ }^{1}$ regarding neck auscultation with keen interest, and have concerns regarding several issues with this technique. Firstly, Dr. Christodolou suggests that neck auscultation, for the purpose of confirming tracheal tube position, may be beneficial for emergency resuscitation in settings outside of the operating room. Most motor vehicle accident casualties requiring tracheal intubation are considered to have potential cervical spine injury contraindicating cricoid pressure. Secondly, the described neck auscultation maneuver requires advancement and withdrawal of the endotracheal tube over a distance of $1-2 \mathrm{~cm}$. In some instances, this maneuver could lead to extubation and ensuing disastrous consequences. Thirdly, this maneuver could also result in mucosal injury when the tip of the endotracheal tube strikes the tracheal mucosa. Furthermore, in addition to the individual performing tracheal intubation and the person responsible for applying cricoid pressure, the maneuver requires an assistant for neck auscultation. This may not be feasible in emergency situations. Finally, auscultation may be difficult in patients with thick necks.

Muhammad Farooq FCARCSI FCPS DA (UK) Coombe Women's Hospital Dublin, Ireland E-mail: muhammadfarooqch@yahoo.com Accepted for publication November 23, 2007.

\section{Reference}

l Christodoulou C. Neck auscultation: a simple new method for confirming tracheal intubation (Letter). Can J Anesth 2007; 54: 854-5.

\section{Reply:}

"There are many hypotheses in science which are wrong. That's perfectly all right; they're the aperture to finding out what's right. To be accepted, new ideas must survive the most rigorous standards of evidence and scrutiny." - Dr. Carl Sagan.

I thank Dr. Faroog for the questions he has raised regarding the technique I have described. Firstly, the application of cricoid pressure to augment the sound emanating from the endotracheal tube striking the cricoid cartilage and tracheal rings is an observation rather than a formal component of the technique. It should never be applied in settings where cricoid pressure is contraindicated. The advance and withdrawal maneuver is a confirmatory sign that need only be applied if doubt exists as to the sounds created by the original passage of the endotracheal tube. Given that many clinicians are involved in the actual intubation, the advance and withdrawal maneuver becomes the sole confirmatory test. The issue of inadvertent tracheal extubation is an important one. Caution should be exercised not to withdraw the endotracheal tube beyond a depth considered to be consistent with subglottic tube placement. A subset of patients may exist in whom the technique is not suitable. Two prospective studies, with an enrolment of over 200 patients, have been completed. The findings should provide further clarity as to the utility of this technique.

The accepted gold standards for confirmation of tracheal intubation are not available to many clinicians in underprivileged settings. A stethoscope is usually always available.

Chris Christodoulou MBChB DA (UK) FRCPC

St. Boniface Hospital, University of Manitoba, Winnipeg, Canada

E-mail: chrischristodoulou@shaw.ca

\section{Rupture de la membraneuse trachéale après intubation par une sonde double lumiève droite [Tracheal rupture after intubation with a right double lumen tube]}

Au rédacteur en chef, La rupture trachéobronchique est une complication rare de l'intubation par sonde double lumière sans éperon $(0,04 \%){ }^{1}$ Nous rapportons le cas d'un patient âgé de 60 ans, programmé pour une résection du lobe supérieur gauche pour tumeur bronchique. 
L'examen pré-anesthésique est sans particularité. Une prémédication à base de $1 \mathrm{mg} \cdot \mathrm{kg}^{-1} \mathrm{~d}$ 'hydroxyzine est instaurée la veille et le matin de l'intervention. Un cathéter péridural est mis en place au niveau D3/D4 avant l'induction de l'anesthésie et l'analgésie est commencée dès lors avec un mélange de bupivacaine $0,125 \%$ et de fentanyl. L'anesthésie générale est réalisée avec l'injection de propofol $2 \mathrm{mg} \cdot \mathrm{kg}^{-1}$, fentanyl $3 \mu \mathrm{g} \cdot \mathrm{kg}^{-1}$, et rocuronium $0,6 \mathrm{mg} \cdot \mathrm{kg}^{-1}$ et la trachée est intubée avec une sonde double lumière Robertshaw $37 \mathrm{CH}$ droite (Broncho-Cath ${ }^{\mathrm{TM}}$ right, Mallinckrodt Medical, Athlone, Irlande) dont la position est vérifiée à l'aide d'un fibroscope. La ventilation unipulmonaire se fait en oxygène pur avec un volume courant à $420 \mathrm{~mL}\left(7 \mathrm{~mL} \cdot \mathrm{kg}^{-1}\right)$ pour une pression de pointe à $25 \mathrm{~cm} \mathrm{H}_{2} \mathrm{O}$, une pression de plateau à $20 \mathrm{~cm}$ $\mathrm{H}_{2} \mathrm{O}$, une fréquence à $14 \mathrm{~min}^{-1}$ et un rapport inspiré/ expiré(I/E) à $1 / 3$. L'entretien de l'anesthésie se fait grâce à l'inhalation de sévoflurane. La stabilité hémodynamique ainsi que la tolérance de la ventilation unipulmonaire est bonne avec une saturation en oxygène se maintenant autour de $98 \%$. L'intervention, d'une durée de $150 \mathrm{~min}$, se déroule sans particularités. Après avoir constaté l'absence de fuite aérienne par les drains, le patient est extubé à la salle de réveil après trois heures d'anesthésie.

Le lendemain de l'intervention, le patient développe un emphysème sous-cutané cervico-facial s'étendant progressivement au thorax et aux deux membres supérieurs et une dyspnée d'aggravation progressive. À la gazométrie artérielle, on retrouve un $\mathrm{pH}$ à 7,32 , une $\mathrm{PaO}_{2}$ à $142 \mathrm{mmHg}$, et une $\mathrm{PaCO}_{2}$ à 43 $\mathrm{mmHg}$. Le diagnostic de rupture trachéo-bronchique post-intubation est suspecté et une bronchoscopie confirme la présence d'une brèche de la membraneuse trachéale de $1 \mathrm{~cm}$ au niveau de son tiers inférieur. Une tomodensitométrie thoracique montre un triple syndrome aérique : pneumomédiastin, pneumothorax et emphysème sous-cutané diffus (Figure). Devant cette brèche de petite taille et non compliquée, nous optons pour une attitude conservatrice associant une oxygénothérapie et un drainage thoracique aspiratif. L'évolution est favorable avec régression de la symptomatologie au jour sept postopératoire et amélioration des gaz du sang : pH à 7,40, une $\mathrm{PaO}_{2}$ à 281 $\mathrm{mmHg}$, et une $\mathrm{PaCO}_{2}$ à $38 \mathrm{mmHg}$. Quinze jours plus tard, une bronchoscopie de contrôle montre une cicatrisation de la plaie, sans séquelle.

Plusieurs facteurs favorisant les plaies trachéales ont été identifiés : le sexe féminin (peut-être à cause d'une membraneuse postérieure plus fine), le mauvais état général, l'âge avancé, le diabète et l'utilisation d'un guide métallique durant l'intubation. De même,

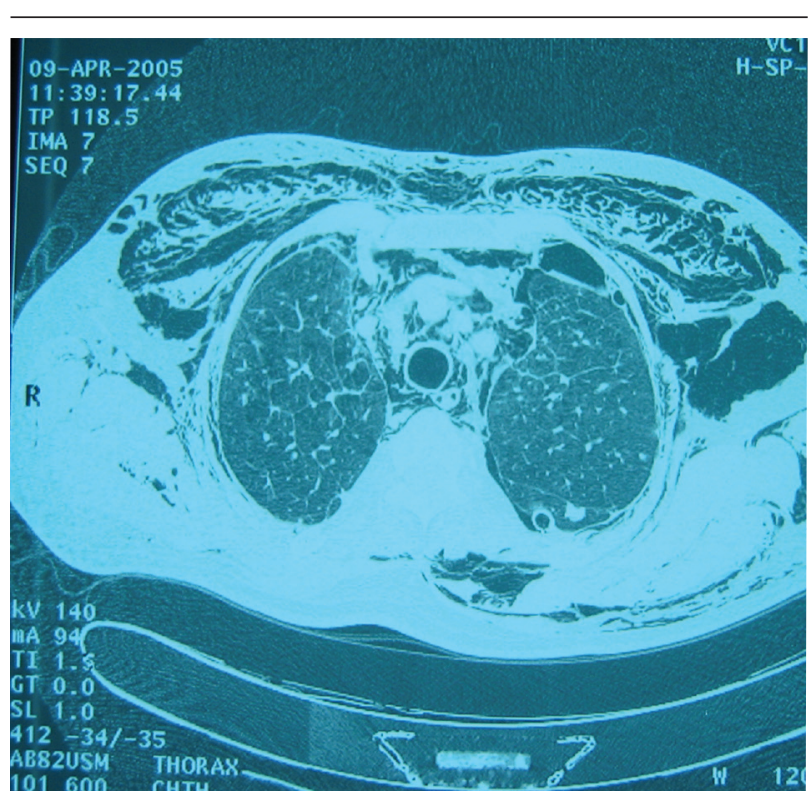

FIGURE Image tomodensitométrique montrant l'emphysème sous-cutané, un pneumothorax gauche et un pneumomédiastin.

l'utilisation de sondes double lumière avec éperon pourrait augmenter le risque traumatique, tout comme une pression excessive du ballonnet dans le cas des lésions des bronches souches. On souligne le rôle potentiellement favorisant d'un accès de toux chez un patient peu sédaté au cours de l'intubation. Chez notre patient, la lésion de la membraneuse du tiers inférieur de la trachée serait due directement à un traumatisme direct au cours de l'intubation. Classiquement, on observe au moins un des signes cliniques évocateurs de plaie de l'arbre bronchique principal : détresse respiratoire, dyspnée, hémoptysie, emphysème sous-cutané, difficultés au cours de la ventilation artificielle. ${ }^{2}$ Les symptômes surviennent après un délai variable : le diagnostic peut être immédiat en per-opératoire, ou n'être apparent qu'après quatre à six heures, voire trois à cinq jours. ${ }^{3}$ Autrefois, le traitement était exclusivement chirurgical, mais un traitement conservateur, dans certains cas, a prouvé récemment son efficacité, comme c'est le cas de notre patient, porteur d'une lésion non compliquée de moins de $2 \mathrm{~cm}$. La chirurgie sera réservée aux lésions sévères d'emblée ou devant une aggravation de la symptomatologie. ${ }^{4}$

\section{Lahcen Belyamani PhD \\ Hassan Kabiri PhD \\ Noureddine Drissi Kamili PhD}


Hôpital Militaire d'Instruction Mohammed V Rabat, Rabat, Maroc, Afrique

Courriel : lbelyamani@hotmail.com

Accepté pour publication le 28 novembre 2007.

\section{Références}

1 Liu H, Jahr JS, Sullivan E, Waters PF.

Tracheobronchial rupture after double-lumen endotracheal intubation. J Cardiothorac Vasc Anesth 2004; 18: 228-33.

2 Hofmann HS, Rettig G, Radke J, Neef H, Silber RE. Iatrogenic ruptures of the tracheobronchial tree. Eur J Cardiothorac Surg 2002; 21: 649-52.

3 Kaloud H, Smolle-Juettner FM, Prause G, List WF. Iatrogenic ruptures of the tracheobronchial tree. Chest 1997; 112: 774-8.

4 Brichet A, Ramon P, Marquette CH. Sténoses et complications trachéales postintubation. Réanimation 2002; 11: $49-58$.

\section{Patient-controlled oral airway insertion to facilitate awake fibreoptic intubation}

\section{To the Editor:}

The main challenge during flexible, fibreoptic, endotracheal intubation is the considerable discomfort and associated gagging that occurs whenever airway topicalization is either difficult or incomplete. Different techniques, including those utilizing Berman intubating airways (Vital Signs, Totowa, NJ, USA), have been developed to enhance upper airway anesthesia. Despite such advances, topicalization of the airway can be a challenging and time consuming procedure. Recently we employed a "patient-controlled" technique using a lidocaine-coated Berman airway to facilitate a distress-free fibreoptic intubation in a patient with a known difficult airway.

The patient, an obese (111 kg), 46-yr-old male scheduled for a repeat hip arthroplasty, experienced difficulties with airway management, after induction of anesthesia for hip debridement two weeks prior. He required a laryngeal mask airway as a temporizing measure following multiple attempts at tracheal intubation by direct laryngoscopy. His surgery was cancelled due to difficulties associated with bag-mask ventilation and a suspicion of aspiration. Three days later, we scheduled a hip debridement and planned an awake, fibreoptic intubation. We administered midazolam $4 \mathrm{mg}$ iv and glycopyrrolate $0.3 \mathrm{mg}$ iv in the operating room; and we achieved topicalization using

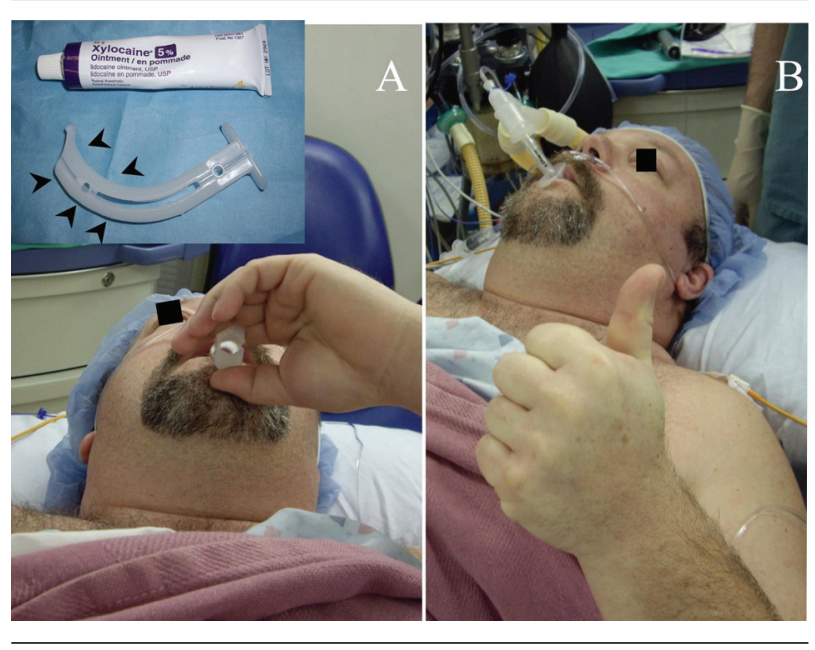

FIGURE The local anesthetic (arrows) is applied to the distal end of the oral intubating airway (Berman, Vital Signs, Totowa, NJ, USA) for effective 'patient-controlled' airway insertion and anesthetic application to the patient's airway. The patient appeared relaxed while he inserted the oral airway (A). This procedure was completed within one minute. The awake, fibreoptic intubation (B) was successful, expedient and tolerated well.

nebulized $4 \%$ lidocaine $(4 \mathrm{~mL})$. An oral bite block was used to aid bronchoscope insertion. Nevertheless, despite a reported lack of history of sensitivity to gagging, the patient was reported to have complained of discomfort from gagging and he also experienced profound coughing fits.

At the time of presentation, examination of the patient's airway revealed a Mallampati grade 3 , a full set of teeth, and reduced neck extension. The anesthetic plan focused on attempting fibreoptic intubation through an intubating airway to ensure adequate topicalization. Routine monitors were applied and an $18 \mathrm{G} i v$ was established. The patient received glycopyrrolate $0.25 \mathrm{mg} i v$, but, at this point, no intravenous sedation was administered. We explained the planned, oral airway insertion technique to the patient and obtained his consent. To begin with, the patient received two sprays of $2 \%$ lidocaine to anesthetize his mouth and oropharynx, then a thin layer of $5 \%$ lidocaine ointment $(12 \mathrm{~mL})$ was applied to the distal portion of the oral airway (Figure, Panel A) which was then progressively and slowly advanced. Within one minute, the patient was easily capable of completely inserting the airway without gagging. Next, $1 \mathrm{~mL}$ of $4 \%$ lidocaine was atomized via the airway, and the vocal cords were visualized immediately upon advancement of the fibreoptic scope. Four millilitres of $4 \%$ lidocaine were then injected through the suc- 